\title{
STRUTTURA GEOLOGICO-SISMICA DELL'EUROPA CEN- TRO-MERIDIONALE, DELL'ITALIA E DEL MEDITERRANEO CENTRO-OCCIDENTALE, QUALE RISULTA DA RECENTI RICERCHE COMPIUTE IN ITALIA (*)
}

\section{Pietro Caloi}

In questa relazione mi propongo di riassumere quanto è stato fatto, negli ultimi anni, da ricercatori italiani sul problema delle caratteristiche geologico-sismiche del continente europeo. Non si tratta quindi di una sintesi di lavori interessanti esclusivamente l'Italia, ma un riassunto delle ricerche compiute da italiani sulle proprietà sismiche dell'Europa, anche se lali ricerche riguardano come è ovvio in modo particolare l'Italia.

Sono stati presi in considerazione soltanto lavori basati sull'interpretazione di registrazioni sismiche, anche se integrati con considerazioni macrosismiche. Non ho preso in esame lavori dedicati esclusivamente all'elaborazione dei dali macrosismici.

Distribuzione geografica delle scosse esaminate. - Per siò che concerne la distribuzione greografica delle scosse esaminate dirò che esse riguardano il Veneto (in numero di 4) $\left({ }^{1 \cdot 2}\right)$, l'alto Adriatico (3) $\left({ }^{3}\right)$, la Valle Padana (1) ( $\left.{ }^{4}\right)$, la Toscana (4) (" $\left.{ }^{5}\right)$, il Lazio ( ${ }^{10}$ ), l'Abruzzo (2) $\left({ }^{11-12}\right)$, lo Jonio - presso le coste calabre $\left({ }^{13}\right)-(1)$ e presso le coste greche - (1) ( $\left.{ }^{14}\right)$, il basso Tirreno (2) $\left(^{15-16}\right)$ e - fuori d'Italia - la Svizzera (2) (17), il Württemberg (13) (17), nonché terremoti extra-europei, studiati nei loro riflessi sulla costituzione del continente europeo, come il terremoto del Turkestan del 2 novembre 1946 e della dorsale mediana atlantica del 24 aprile 1947.

Non mi soffermerò sulla sismicita - intesa come frequenza $e$ intensitia delle scosse - delle zone sismiche interessate dai terremoti oggetto dei lavori qui sintetizzati: l'esame di tale caratteristica esula dai limiti di questa breve relazione.

Qui si vuole solo accennare come, dallo studio dei terremoti citati, si siano potuti trarre alcuni elementi, atti a proporre - con buona

(*) Comunicarione presentata al Convegno della «Commission Séismologiqu: Européenne 》 (Stoccarda, 22-27 Settembre 1952). 


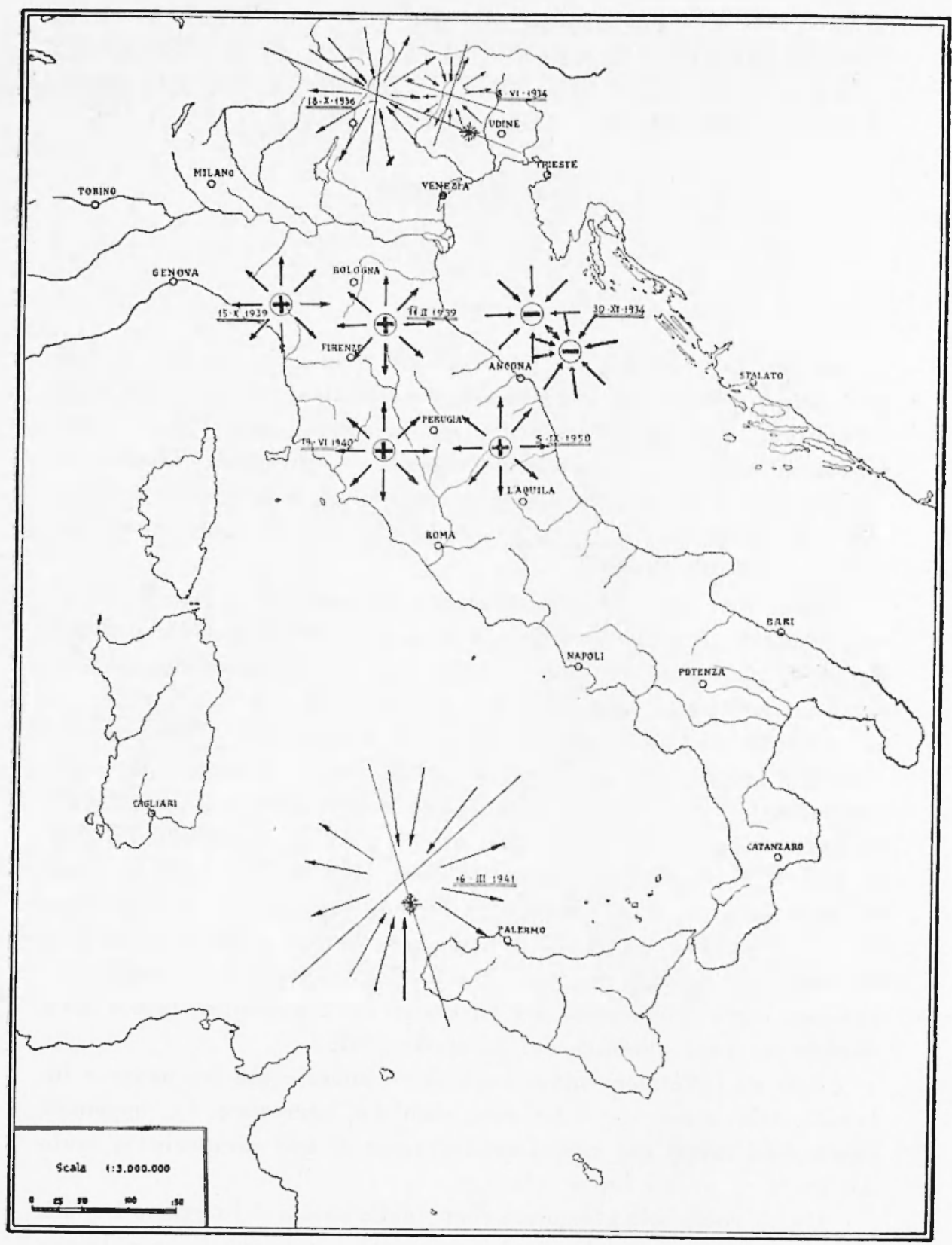

Fig. 1 
approssimazione - alcune conclusioni sui valori delle velocità delle onde longitudinali e trasversali, sulla stratificazione profonda del continente europeo, sulle radici delle Alpi e degli Appennini, sul meccanismo di produzione dei terremoti per ciò che riguarda in particolare la zona delle Alpi, l'alto Adriatico e la zona degli Appennini.

Velocità di propagazione delle onde longitudinali e trasversali. Sono stati rivelati, in modo chiaro, tre tipi distinti di onde longitudinali e trarsversali, corrispondenti a tre diversi mezzi di propagazione: il mezzo ultrabasico, iniziante il mantello terrestre, il mezzo hasaltico (o peroditico, o del gabbro) e il mezzo del "granito". Non è esclusa l'esistenza di onde interessanti altri mezzi di diversa natura geologica; ma, allo stato attuale delle ricerche, non è dato di poter trarre nessuna conclusione al riguardo.

Indicando con $P n, P^{*}, P g$ le onde longitudinali interessanti i tre mezzi nell'ordine detto e con $S n, S^{*}, S g$ le corrispondenti onde trasversali, ecco i valori delle corrispondenti velocità nei tre mezzi considerati :

\begin{tabular}{|c|c|c|c|c|c|c|}
\hline . & $\begin{array}{c}\text { Vinn } \\
\mathbf{K m} \mid \text { Hec }\end{array}$ & $\mathrm{V}^{* *}$ & $V_{\mathrm{Pg}}$ & Vin & $\mathrm{VH}^{*}$ & V $\mathrm{sg}$ \\
\hline $\begin{array}{l}\text { Regione renefa } \\
\text { epicentro Cansiglio: }\end{array}$ & & & & & & \\
\hline 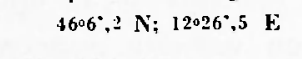 & $8,00 \div 0,01$ & $6,61 \div 0,04$ & $5,2 \div 0,04$ & $4,11 \pm 0,01$ & 3,6, & $3,36+0,025$ \\
\hline $\begin{array}{l}\text { Alto Adriatico } \\
44 \circ 18^{2}, 1 \mathrm{~N} ; 13^{\circ} 29^{\circ}, 6 \mathrm{E}\end{array}$ & & & & & & \\
\hline $\begin{array}{l}\text { Regione Toscana } \\
41^{3} \cdot 4 \text { N: } 11^{1038}, 7 \text { E }\end{array}$ & $2,86+0,04$ & - & $5,2-5,4$ & $4,34 \nmid 0,02$ & & $3,01 \div 0,04$ \\
\hline $44014.0 \mathrm{~N} ; 10 \times 12^{\circ}, 2 \mathrm{E}$ & $8,16.1 .0,06$ & $6,63 \div-0,08$ & $5,25 \div 0.00$ & $1,44 \div 0,02$ & $3,57 \div 0,04$ & $3,06 \div 0,1$ \\
\hline 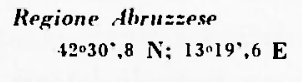 & $8,19 \div 0,04$ & $6,38 \div 0.05$ & $5,46 \div 0.18$ & $4,39 \div 0,02$ & & $3,01 \div 0,02$ \\
\hline $\begin{array}{l}\text { Regione Laziale } \\
\qquad 42 \times 1,85 \mathrm{~N} ; 13^{04}, 55 \mathrm{E}\end{array}$ & 8,09 & - & 5,39 & 4,40 & - & 3,05 \\
\hline $\begin{array}{l}\text { Regione Calabra } \\
\qquad 38041^{\circ}, 4 \mathrm{~N} ; 16047^{\circ}, 7 \text { E }\end{array}$ & $7,94- \pm 0,18$ & $6,96 \div 0,41$ & - & $4.5 \div \div 0.08$ & $1,00 \div 0,09$ & - \\
\hline
\end{tabular}

Una ricerca sul terremoto del basso Tirreno del 16 marzo 1941 ha messo in evidenza un notevole anticipo nei tempi di registrazione delle onde di dilatazione e di distorsione nelle stazioni più vicine all'epicentro. Analoghe osservazioni sono state fatte nello studio del 
terremoto profondo delle isole Lipari del 13 aprile 1938, studio che è stato ripreso recentemente da Caloi e Giorgi. Vedremo più oltre che tale notevolissima anomalia può trovare la sua spiegazione nella natura vulcanica della zona.

Un'altra considerevole anomalia che risulta dall'osservazione della tabella riportata consiste nell'elevato valore assunto dalla velocità delle onde trasversali destate dal terremoto dell'alto Adriatico del 30 novembre 1934 , in una zona caratterizzata da terremoti di sprofondamento. Un'analoga anomalia è stata messa in evidenza da P. E. Valle per lo Jonio, dove, come per l'alto Adriatico, ad una leggera diminuzione della velocità delle onde $P \boldsymbol{n}$ fa riscontro un sensibile aumento della velocità delle onde $S n$. Se indichiamo con $\lambda$ e $\|$ le costanti di Lamé e con e la densità del mezzo, nel tentativo di spiegare detta anomalia, P. E. Valle è condotto alla relazione $\left({ }^{13}\right)$

$$
\frac{\left|d_{u}\right|}{\iota}<-\frac{\mid d_{0} !}{\varrho}<-\frac{|d \lambda|}{\wedge},
$$

che deve valere affinché nel passare dal continente al Mediterraneo si osservi una diminuzione della velocità delle onde $P_{n}$ ed un aumento della velocità delle onde $S n$.

Sempre in tema di velocità di propagazione delle onde sismiche, in un ampio studio su diciassette terremoti centro europei è stato possibile trovare che, nello strato di $20 \mathrm{~km}$ di "granito " - costituente la prima stratificazione della crosta per l'Europa centrale - le onde longitudinali e trasversali dirette si propagano con velocità crescenti con la profondità, secondo le leggi ( $\left.{ }^{17}\right)$

$$
V_{\mathrm{Pg}}=5,530(1+0,00158 . h) ; V_{\mathrm{SB}}=3,275(1+0,00158 . h)
$$

per $\sigma=0,23$.

Stratificazione della crosta terrestre. - Gli spessori degli strati, costituenti la crosta terrestre, sono stati ottenuti con metodi basati o sulla propagazione delle onde spaziali, o sulla dispersione delle onde superficiali o sulla variazione del fattore

$$
k=\frac{v_{\mathrm{p}} \cdot v_{\mathrm{s}}}{v_{\mathrm{p}}-v_{\mathrm{s}}} .
$$

Le prime determinazioni riguardano lo spessore dello strato delle onde $\mathrm{Pg}$ in corrispondenza delle prealpi Carniche e del Cansiglio. Si sono ottenuti, in diverse determinazioni, valori variabili fra $40 \mathrm{~km}$ 
e $34 \mathrm{~km}$ e si è concluso che lo spessore dello strato delle $P g$, in corrispondenza del sistema alpino orientale ̀̀ di $35 \mathrm{~km}$. ca.

$\mathrm{Si}$ è poi passati all'Italia centrale e precisamente all'Appennino tosco-romagnolo e alle Alpi Apuane. Lo spessore dello strato del " granito " è risultato, rispettivamente, di $25 \mathrm{~km}$ e di $30 \mathrm{~km}$. In corrispondenza del Gran Sasso, determinazioni successive lianno portato ad un valore di $25 \mathrm{~km}$, che sembra essere lo spessore medio del sistema degli Appennini. Altre ricerche fanno ritenere che, in corrispondenza della Valle Padana lo spessore dello strato del "granito " sia alquanto minore (dell'ordine di $10 \mathrm{~km}$ ).

Nel 1943 fu provato, per la prima volta clie, in una vasta zona oltre il sistema alpino, comprendente almeno parte della Svizzera e il Wiirttemberg $\left({ }^{17}\right)$, la prima superficie di discontinuità nell'interno della crosta terrestre si trova ad una profondita di circa

$20 \mathrm{~km}$

valore successivamente confermato da altri ricercatori, quali Rothé, Peterschmitt e Wanner.

Il valore ottenuto nel 1943 provava che la superficie di discontinuità fra "granito " e basalto era, per la regione considerata, alquanto più sollevata di quanto non fosse fino allora ritenuto.

Ulteriori determinazioni, condotte con metodi basati sulla dispersione delle onde superficiali, hanno confermato per l'Italia un valore medio di $26 \mathrm{~km}$ per lo strato del "granito", mentre tale strato, nella direzione Jonio-Spagna (interessante gran parte del Mediterraneo) ha un valore medio di 15 , nella direzione Jonio-Adriatico di $17 \mathrm{~km}$, lo spessore dello strato del "granito " nella direzione Turkestan-Roma ¿̀ dell'ordine di $18 \mathrm{~km}\left({ }^{18}\right)$. Ai margini del continente, dal lato dell'Atlantico, tale strato sareblue pressoché mancante $\left(^{20}\right)$.

Risultava evidente che la superficie limitante lo strato del granito non poteva essere parallela alla superficie della Terra: essa appariva sprofondarsi in corrispondenza dei sistemi di montagne.

Con un metodo che non è qui il caso di riferire, nel 1940 ( $\left.{ }^{22}\right)$ fu infatti messo in evidenza clue la prima superficie di discontinuità tende a sollevarsi quando si procede dall'interno del sistema alpino verso Sud e verso Nord. In corrispondenza del centro del sistema alpino (Tirolo), si è trovato la profondità di $40 \mathrm{~km}$, profondità clıe ai margini meridionali (Cansiglio) si riduce a $34 \mathrm{~km}$. Restava cosi provata l'esistenza delle " radici " delle montagne. Tale esistenza è stata con- 
fermata da uno studio successivo di Gutenherg per la Sierra Nevada (California) e, di recente, - con il metodo di Caloi su accennato da Di Filippo e Marcelli per gli Appennini.

Per il sistema alpino, lo strato del "basalto ) (o "gabbro ") ̀̀ risultato piuttosto piccolo, dell'ordine di $11 \mathrm{~km}$, mentre sembra assumere valori alquanto più grandi in corrispondenza degli Appennini.

Natura delle scosse allipocentro e profondità ipocentrali. - Per quanto si riferisce al meccanismo di produzione dei terremoti studiati, va subito detto cbe esso si è esplicato secondo tre diversi modelli: frattura (movimento del suolo dello stesso segno in quadranti opposti), sprofondamento (dilatazioni entro una circonferenza di raggio determinato, compressioni all'esterno), sollevamento (compressioni nell'interno di una circonferenza di dato raggio, dilatazioni all'esterno).

Il primo modello (frattura con mutuo scorrimento di strati) è stato osservato in tutto il sistema alpino, dove esso sembra predominare: l'esempio più caratteristico è stato fornito dal terremoto del Cansiglio del 18 ottobre $1936\left(^{1}\right)$; dovuto a frattura provocata da una coppia di forze inclinate, giacente in un piano pure inclinato.

I terremoti dell'alto Adriatico finora studiati presentano invece il modello caratteristico dei terremoti di sprofondamento. A questo riguardo, è interessante osservare il parallelo nei fenomeni bradisismici della regione. E noto infatti che, in ère geologiche passate, fu molto accentuato il bradisismo negativo in tutta l'Istria e la Dalmazia; e dell'entità di questo bradisismo si hanno chiare testimonianze (forma frastagliatissima della costa adriatica orientale, gran numero di isole presso le coste, ecc.).

L'Appennino presenta invece il fenomeno contrario. Lo studio dei terremoti dell'Appennino tosco-romagnolo, della Garfagnana, del Monte Amiata e, più recentemente, del Gran Sasso d'Italia, ha messo chiaramente in luce il carattere di un sollevamento di strati.

La geologia concorda con queste constatazioni; fra l'altro, l'esame delle fronti di alcune faglie testimonia a favore dei sollevamenti, quali ad esempio quello del Sirente e quello della catena del Gran Sasso verso Campo Imperatore.

Nel Tirreno meridionale i terremoti studiati rientrano invece nel modello caratteristico delle fratture. Qui però il fenomeno presenta un interesse a sé, trattandosi di terremoti di orig̣ine profonda.

A proposito della profondità ipocentrale, va osservato che i terremoti interessanti il sistema alpino, in senso lato, riguardano esclu- 
sivamente la crosta terrestre; molti fra essi originano a piccole profondità ipocentrali. Di 17 terremoti interessanti detta zona, 7 hanno avuto profondità inferiori ai $10 \mathrm{~km}, 6$ inferiori ai $20 \mathrm{~km}$ ed 1 solo ebbe origine alla base della crosta terrestre.

I terremoti dell'alto Adriatico sembrano originare da maggiori profondita: probabilmente ai limiti della crosta terrestre.

Le scosse che avvengono nell'ambito degli appennini originano invece, quasi esclusivamente, a piccola profondità, inferiore ai $10 \mathrm{~km}$.

Mi sembra di estremo interesse a questo riguardo, dare il dovuto risalto anche all'altro particolare che distingue la sismicità dell'Adriatico da quello della catena, ad esso parallela, degli Appennini: l'opposto meccanismo da cui originano i terremoti nelle due zone contigue: agli sprofondamenti in profondità dell'alto Adriatico, fanno riscontro i sollevamenti superficiali della catena appenninica.

Sembra quasi che alle compressioni laterali determinati dai primi conseguano i sollevamenti causati dai secondi. A meno che le qualifiche di causa ed effetto non debhano essere invertite. Ad ogni modo, siamo in presenza di un aspetto grandioso dell'orogenesi ancora in atto nella regione in questione, come è confermato da molti altri indizi di carattere geologico.

Considerazioni a parte merità la sismicità del hasso Tirreno. Qui molti terremoti originano a grandi profondità: quello del 16 marzo 1941 ad una profondità di circa $85 \mathrm{~km}\left({ }^{15}\right)$ e quello delle isole Lipari del 13 aprile 1938 alla profondità di $300 \mathrm{~km}$ circa (16).

La zona si presenta notevolmente anomala da tutti i punti di vista: geologico, geodetico, sismico. A Nord e a Sud della Sicilia, oltre che sull'isola stessa, sono numerose le tracce di vulcanismo attivo o spento: l'isola di Ustica, d'origine vulcanica, il gruppo insulare delle Lipari di recente origine vulcanica, con i due vulcani attivi dello Stromboli e dell'isola di Vulcano, un vulcano spento trovasi a Sciacca, Pantelleria è d'origine vulcanica: tutta la zona insomma, senza citare i vulcani maggiori, è stata spesso teatro di eruzioni vulcaniche sottomarine, rivelate da terremoti e da maremoti.

Abhiamo accennato alla grande anomalia sulla propagazione delle onde sismiche provocate dai terremoti profondi citati e rivelata dal notevole anticipo nella registrazione delle onde $P n$ ed $S n$ negli osservatori vicini alla zona epicentrale.

Poiché le onde trasversali danno deboli registrazioni nella zona, si è portati a ritenere che le maggiori velocità non siano da attribuire 
ad una maggiore rigidità del mezzo, bensì ad un difetto di densità da collegarsi alle enormi riserve di materiale vulcanico. Un contributo in questo senso può dare pure la sottigliezza dello strato superficiale del "granito" cbe, nella zona, sembra pressoché mancante.

Strettamente collegata alla notevole anomalia sismica considerata sta il fatto cbe la regione in parola è sede di una cospicua anomalia gravimetrica positiva.

$$
\text { Roma - Istituto Nazionale di Geofisica - Ottobre } 1952 .
$$

\section{RIASSUNTO}

Si riassumono $i$ risultati principali ottenuti da ricercatori italiani, negii ultimi quindici anni, nello studio delle caratteristiche geologichesismiche della penisola italiana, del Mediterraneo centrale e di una vasta zona comprendente la Svizzera settentrionale e il Württemberg, nonché di zone marginali atlantiche.

I. - Lo spessore dello strato delle onde $\mathrm{Pg}$ per le Alpi orientali, meridionali (Cansiglio e Prealpi carniche) è risultato di circa $35 \mathrm{~km}$.

II. - Lo spessore dello strato delle $\mathrm{P}^{*}$, per la stessa zona, $\grave{e}$ dell'ordine di $10.15 \mathrm{~km}$.

III. - Le Alpi presentano "radici»: nella parte centrale del sistema alpino, tali radici sembrano raggiungere i $40 \mathrm{~km}$.

IV. - In corrispondenza della Val Padana lo spessore del a granito" sembra diminuire sensibilmente.

V. - Lo strato delle $\mathrm{Pg}$ torna ad ispessirsi in corrispondenza dell'Appennino.

VI. - Anche il sistema degli Appennini presenta "radici». Lo spessore di tale sistema, di circa $25.30 \mathrm{~km}$ in corrispondenza del suo asse centrale, tende ad assottigliarsi verso l'Italia meridionale: in corrispondenza della Sicilia sembra assai sottile.

VII. - Lo spessore medio dello strato del " granito " per il Mediterraneo centro-occidentale risulta di $15 \mathrm{~km}$ circa.

VIll. - In una vasta zona dell'Europa centrale, comprendente la Svizzera settentrionale e il Württemberg, lo strato del "granito" presenta uno spessore di soli $20 \mathrm{~km}$, come risulta dallo studio di $13 \mathrm{ter}$ remoti interessanti la zona, compiuto sul 1943. Tale spessore tende ad aumentare procedendo verso il sistema alpino.

IX. - Nella stessa zona, di cui al n. VIll, la velocità delle onde longitudinali e trasversali dirette segue le seguenti leggi: 


$$
V_{\mathrm{Ph}}=5,530(1+0,00158 . \mathrm{h}) \quad ; \quad V_{\mathrm{sg}}=3,275(1+0,00158 . \mathbf{h}),
$$

per lo variabile da 0 a $20 \mathrm{~km}$.

$X$. - La velocità di propagazione delle onde $\mathbf{P g}_{\mathrm{g}}$ nell'Italia peninsulare risulta, in media, di $5,4 \mathrm{~km} / \mathrm{sec}$; alquanto inferiore a quella osservata per l'Europa centrale di $5,7 \mathrm{~km} / \mathrm{sec}$ in media.

$X I$. - Per ciò che riguarda la profondità ipocentrale, questa presenta in genere piccoli valori nel sistema alpino, dove, per altro, si possono verificare terremoti con profonditá fino a $30 \mathrm{~km}$ circa. Nell'Appennino, le profondità ipocentrali sono quasi esclusivamente comprese fra 0 e $10 \mathrm{~km}$ : molto piccole quindi.

Nel Tirreno meridionale, invece, oltre ai terremoti d'origine vulcanica con ipocentro prossimo alla superficie, si verificano terremoti di notevole profondità ipocentrale, variabile fra 100 e $300 \mathrm{~km}$.

$X I I$. - Un interesse particolare presenta la natura fisica delle scosse all'ipocentro. Si distinguono - a questo riguardo - tre zone: la zona delle $A l_{p i}$, dove la distribuzione superficiale delle compressioni e delle dilatazioni è, generalmente, quella propria delle fratture, a zona del medio Adriatico, caratterizzata da violente scosse dovute a sprofondamento di strati e la zona degli Appennini, dove si verificano, quasi esclusivamente terremoti presentanti il modello proprio dei sollevamenti.

Le zone dell'Adriatico e della catena degli Appennini, che corrono geograficamente parallele l'una all'altra, sembrano quindi animate, in profondità, da movimenti in senso contrario.

Sarà interessante stabilire se esistono, tra questi movimenti, rapporti di causa ad effetto.

XIII. - La zona del basso Tirreno è caratterizzata da scosse del tipo a frattura. Essa è fortemente anomala sia per il vulcanismo in atto, sia per $i$ terremoti profondi di cui è sede, sia per il notevole anticipo con cui le onde longitudinali e trasversali - destate da questi terremoti - vengono registrate nelle stazioni sismiche circonvicine: queste anormalità trovano pure riscontro in una notevole anomalia gravimetrica positiva.

$X I V$. - I risultati sopra riassunti non hanno naturalmente carattere definitivo: essi possono subire modifiche più o meno profonde con l'affinarsi dei metodi di ricerca. 


\section{SUMMARY}

We summarize here the principal results obtained by Italian researchers in the last 15 years from the study of the geologic and seismic characteristics of the Italian peninsula, covering the central Mediterranean and a vast zone which includes Northern Switzerland and Würtemberg, and also border zones of the Atlantic.

I. - The thickness of the layer of the $\mathrm{Pg}$ waves for the Eastern Alps and for the southern Alps (Cansiglio and Prealpi carniche) is about $35 \mathrm{~km}$.

II. - The thickness of the layer of the $\mathrm{P}^{*}$ waves for the same zone is on the order of $10-15 \mathrm{~km}$.

III. - The Alps have aroots : in the central part of the Alpine system such roots seem to reach to about $40 \mathrm{~km}$.

IV. - In comparison with the Val Padana the thickness of the “granite» seems sensibly less.

$V$. - The layer of $\mathrm{Pg}$ becomes again thick under the Apennines.

VI. - Also the Apennines system has a roots ». The thickness of this system of mountains, on the order of $25-30 \mathrm{~km}$ in comparison with its central axis, tends to diminish toward southern Italy: in comparison with Sicily it seems rather thin.

VII. - The mean thickness of the layer of granite for the centralwestern Mediterranean is about $15 \mathrm{~km}$.

VIlI. - In a large zone of central Europe, including northern Switzerland and Württemberg, the layer of aranite $\$$ has a thickness of only $20 \mathrm{~km}$, as computed from the study of 13 earthquakes pertaining to the zone, in 1943. This thickness tends to increase in the direction of the Alpine system.

$I X$. - In the same zone (that of VIII) the velocities of the direct longitudinal and transverse waves follow the following law:

$$
V_{\mathrm{Pz}}=5,530(1+0,00158 . \mathrm{h}) \quad ; \quad V_{\mathrm{Sq}}=3,275(1+0,00158 . \mathrm{h}),
$$

for $\mathrm{h}$ variable from 0 to $20 \mathrm{~km}$.

$X$. - The velocity of propagation of the $\mathrm{Pg}$ waves in peninsular Italy is, on the average, $5,4 \mathrm{~km} / \mathrm{sec}$; rather less than that observed for central Europe of about $5,7 \mathrm{~km} / \mathrm{sec}$.

$X I$. - As regards the hypocentral depth one has, in general, small values in the Alpine system where, nevertheless, one can ascertain earthquakes with depths up to about $30 \mathrm{~km}$. In the Apennines the 
hypocentral depths are almost exclusively in the range from 0 to 10 $\mathrm{km}$, thus very small. In the southern Tirreno instead, in addition to earthquakes of volcanic origin with the hypocenter close to the surface, one finds earthquakes of notable hypocentral depth, variable from 100 to $300 \mathrm{~km}$.

XII. - Of particular interest is the physical nature of the shocks at the hypocenter. For this purpose one distinguishes three zones: the Alpine zone, where the surface distribution of the compressions and dilatations is generally that properly of the fractures; the central Adriatic zone characterized by violent shocks due to sinking of the layers; and the zone of the Apennines where one finds almost exclusively model earthquakes of the raising kind.

The zones of the Adratic Sea and the Apennines chain, which run geographically parallel to each other, seem to be brought about, as regards the depth, by opposite movements.

It would be interesting to establish if there exists a relation of rause to effect between these movements.

XIII. - The zone at the base of the Tirreno is characterized by shocks of the fracture type. It is strongly anomalous either hecause of volcanic activity, or because of the deep earthquakes of which it is the seat, or because of the notable advance with which the longitudinal and transverse waves, set in motion by these earthquakes, are registered in the nearby surrounding seismological stations. This anomalous behavior even finds confirmation in a notable positive gravimetric anomaly.

XIV. - The results summarized above are naturally not of a definite character and they can be modified more or less seriously with refinements in the methods of research.

\section{BIBLIOGRAFIA}

(1) Calor P., Ricerche su terremoti ad origine vicina. Scosse del Cansiglio del. l'ottobre 1936.XVI. La Ricerca Scientifica, IX, 7.8 (1938).

(2) Calor P. Attività sismica in ltalia nel decennio 1930.1939. Le Monnier, Fi. renze (1942).

(3) Calol P., Il terremoto adriatico del 30 novembre 1934. Boll. Soc. Sismol. It, XXXV (1937).

(4) Calol Pa Epicentro e profondità ipocentrale del terremoto di Salò del 24 gennaio 1943. Istituto Veneto di Soc., Lett. ed Art, CIV (1944-45). 
(5) Calor P., Il terremoto dell'Appennino tosco-romagnolo dellil febbraio 1939-XVII. La Ricerea Seientifica, X (1939).

(i) Calor P., Caratteristiche sismiche dell'Appennino tosco-romagnolo. La Ricerca Scientifica, XI (1940).

(7) Rosini E., Il terremoto della Garjagnana del 15 ottobre 1939. La Ricerca Scientifica, XI (1940).

(8) Di Filippo D, Il terremoto del Monte Amiata del 19 giugno 1940. La Ricerca Scientifica, XII (1941).

(9) Gıono $\mathrm{Ml}$, Il terremoto del Monte Amiata del 16 ottobre 1940. La Ricerca Scientifica, XII (1941).

(10) DI FiLippo D, Il terremoto di Cervara di Roma dell'8 settembre 1941. Boll. Soc. Sismol. It., XL (1942).

(11) Dr Filippo D, Il terremoto di Teramo del 29 gennaio 1943. Annali di Geofisica, II (1949).

(12) Di Filippo D. - Marcelli L., Uno studio del terremoto del Gran Sasso d'Italia del 5 settembre 19.50. Annali di Geofisica, IV (1951).

(13) Valte P. E., Contributo allo studio delle caratteristiche sismiche del Mediterraneo centro-orientale. Annali di Geofisica, I (19.18).

(14) Festa C. - Valle P. E, Ina valutazione dello spessose dello strato del "granito ) nel Mediterraneo centro-occidentale. Annali di Geofisica, I (1948).

(1ii) Di FILIPpo D, Studio microsismico del terremoto del basso Tirreno del 16 marzo 1941. Boll. Soc. Sismol. It., XXXIX (1941).

(16) Calor P. - Grongi M, Studio del terremoto delle isole Lipari del 13 aprile 1938. Annali di Geofisica, IV (1951).

(17) Calor P, Caratteristiche sismiche fondamentali dell'Europa centrale. Boll. Soc. Sismol. It, XL (1942).

(18) Peronaci F., Determinazione del valore medio deilo spessore dello strato di granito per i tragitti Turkestan-Europa. Annali di Geofisica, III (1950).

(19) Marcelli L. - Pannocchia G, Terremoto della cresta mediana atlantica del 24 aprile 1947. Annali di Geofisica, I, 4 (1948).

(20) Caloi P. - Marcelli L. - Pannocchia G., Sulla velocità di propagazione delle onde superficiali in corrispondenza dell Atlantico. Annali di Geofisica, II, 3 (1949).

- ID., Ancora sulla velocità di propagazione delle onde superficiali in corrispon. denza dell'Atlantico. Annali di Geofisica, III, 2 (1950).

(21) VAl Le P. E, Sulla struttura della crosta terrestre nel Meditcraneo centrooccidentale. Annali di Geofisica, IV (1951).

(22) Carou $P_{n}$, Sulla velocità di propagazione delle onde $P$ s e sullo spessore dello strato del granito nell'Europa centrale. La Ricerca Scientifica, XI, 11 (1940). 